\title{
Reduction of Cavity-to-Cavity Power/Ground Noise Coupling through Plane Cutout in Multi- layer PCBs
}

\author{
Junwoo Lee, Myunghoi Kim, and Joungho Kim \\ Terahertz Interconnection and Package Laboratory \\ Department of EECS, KAIST \\ 373-1 Gusong-dong, Yusong-gu, Daejeon, Korea \\ fluxus@eeinfo.kaist.ac.kr, teralab@ee.kaist.ac.kr
}

\author{
*Mihai Dragos Rotaru, and *Mahadevan K Iyer \\ * Institute of Microelectronics, \\ 11 Science Park Road, Singapore Science Park II, Singapore
} 117685.

\begin{abstract}
Power/ground noise excited in a plane cavity can be coupled to neighboring plane cavity through a cutout. The coupled noise is substantial at the cavity resonance. This paper addresses the reduction method of the coupled noise through a cutout based on simulation and measurement. We demonstrated that the coupled noise can be suppressed by placing ports (devices) at the proper locations or designing the size of the plane cavity carefully.
\end{abstract}

Keywords-component; power/ground noise, cutout, SSN, noise coupling, Multi-layer PCBs

\section{INTRODUCTION}

The development of semiconductor device technology brings faster operating speed, higher average currents, and lower supply voltage. As a result, the design of low impedance and low noise power/ground distribution networks for highspeed packages and printed circuit boards (PCBs) has become a major challenge due to significant power/ground switching noise [1]. The simultaneous switching noise (SSN) significantly degrades the signal integrity of high speed devices or systems. The SSN excited in the power/ground planes causes the power/ground voltage level at a position in a plane cavity to fluctuate, propagates to other positions by electromagnetic wave propagation in the plane cavity and is also coupled to neighboring plane cavities through cutouts or through other interconnection structures, including the power/ground via. The authors have proposed the analytical model of the SSN coupling between power/ground plane cavities through cutout in multi-layer PCBs and verified it by experiment [2], [3]. According to the analysis, substantial SSN coupling occurs between plane cavities, through a cutout, at resonant frequencies of plane cavities.

In this paper, we proposed a reduction method of SSN coupling between plane cavities through a cutout. Proper positioning of the cutout and the devices at each plane cavity achieves significant noise suppression at certain resonant frequencies. Alternatively, the SSN can be reduced when the frequency components of the SSN generating chip has no correlation with the resonant frequencies of the plane cavity determined by a cavity size and dielectric material. We used a four-layer and two-cavity power/ground structure on a PCB for the analysis and measurement to demonstrate the SSN suppression effect. The coupled noise suppression effect is demonstrated in terms of the transfer impedance at the resonant frequencies, and the time-domain SSN waveforms by changing the locations of the noise source and the noise receiver, and changing switching frequency of the SSN generating chip. The suggested noise suppression method can be usefully adopted in high-performance package and PCB design.

\section{REDUCTION OF CAVITY-TO-CAVITY SSN COUPLING THROUGH A PLANE CUTOUT}

\section{A. Frequency Domain Analysis}

To suppress the coupled SSN through a cutout, we adopted port optimization method introduced by Lei [4]. The suppression effect of the optimal positioning method was demonstrated by simulation using an analytical model [3]. To -verify the method by experiment, test vehicles as shown in Fig. 1 were fabricated.

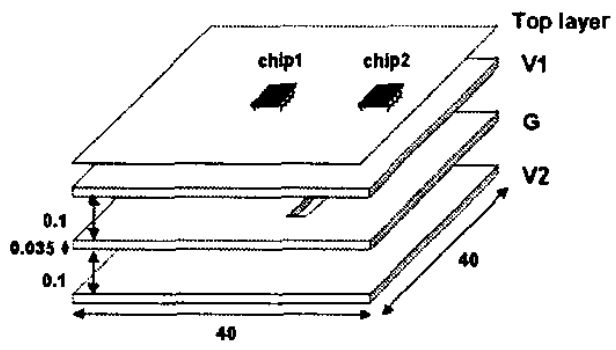

(a)

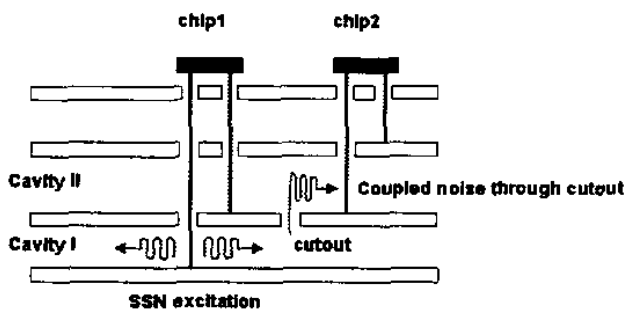

(b)

Figure 1. Structure of the test vehicles. a) 3D view (b) Cross section 


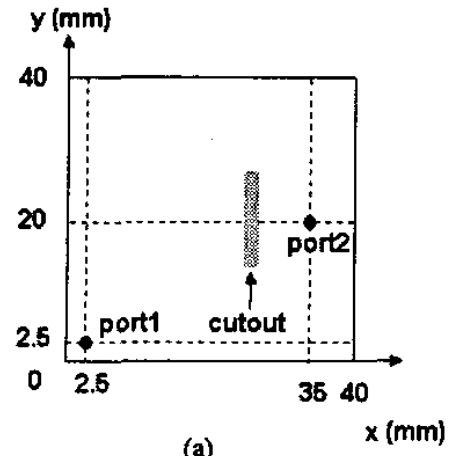

(a)

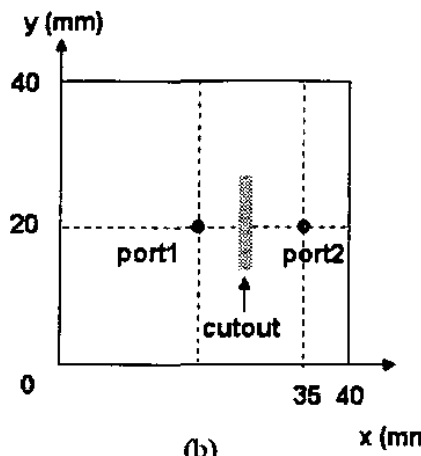

(b)

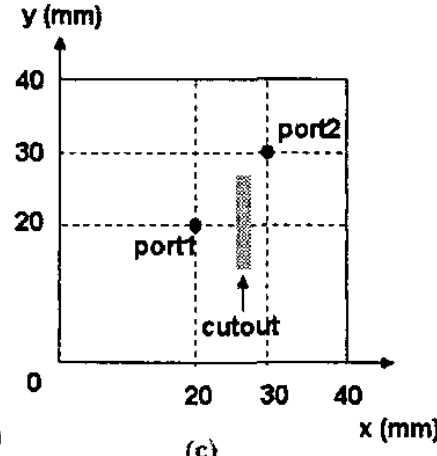

(c)

Figure 2. Port location configurations for the test vehicles: (a) port1 locates at $(2.5,2.5)$ and port2 locates at $(35,20)(b)$ port1 locates at $(20,20)$ and port2 locates at $(35,20)$ (c) port1 locates at $(20,20)$ and port2 locates at $(30,30)$.

The chips 1 and 2 in Fig. 1 correspond to the ports 1 and 2 in the test vehicles, respectively. The port locations are the same as those shown in Fig. 2. The cutout locates at $x=27.5$ $\mathrm{mm}$, and $\mathrm{y}=20 \mathrm{~mm}$ with a $0.5 \mathrm{~mm} \times 10 \mathrm{~mm}$ size. Fig. 3 shows the measured transfer impedance for the sample shown in Fig. 2(a). All the modal impedances except the odd-numbered $n$ can be seen. In Fig. 4, the dotted line represents the measured transfer impedance for the sample of Fig. 2(b) and the solid line is for the sample of Fig. 2(c). The measurements show the same trend as the simulation results in [3], which demonstrates that the optimal positioning method suppresses the coupled noise between the plane cavities through the cutout. Measurement confirmed that all the modal impedances for oddnumbered $m$ or $n$ are suppressed by repositioning chip 1 to the center of the plane and that the even-numbered modal impedances are suppressed further by the positioning of chip 2 . We conclude that the noise coupling between plane cavities through the cutout can be reasonably suppressed by simply placing the chips and the cutouts at proper locations.

\section{B. Time Domain Analysis}

For the time domain evaluation of the optimal positioning method, we conducted transient simulation using measured Sparameters. Consider the test vehicles shown in Fig. 1 and assume that chip 1 has a $1 \mathrm{~A}$ current source. The current source has a $0.65 \mathrm{~ns}$ period with rise and fall times of $75 \mathrm{ps, \text {and }}$ switches for two clock cycles as shown in Fig. 5.

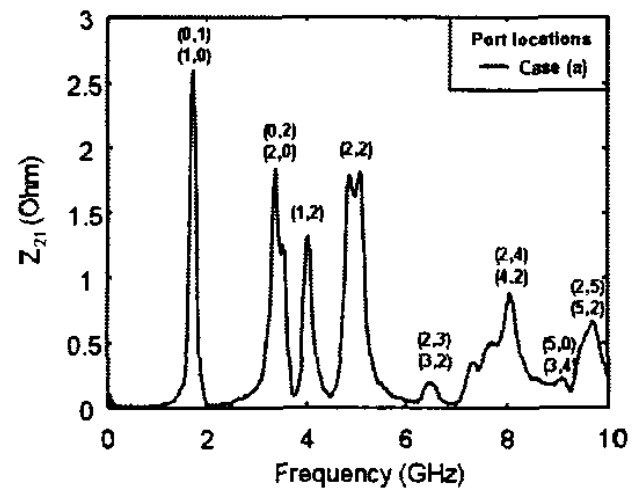

Figure 3. Measured transfer impedance for the test sample shown in Fig $2(a)$; peaks are annotated with the dominant mode numbers $(m, n)$
Then, the current source generates switching noise in the lowest plane cavity $I$ and the noise is coupled into the neighboring plane cavity II through the cutout. The coupled noise can affect the signal integrity of chip 2 as well as the signal integrity of chip 1 .

Fig. 6(a) shows the time domain coupled noise waveform when chip 1 is located at $x=2.5 \mathrm{~mm}, y=2.5 \mathrm{~mm}$ and chip 2 is at $x=35 \mathrm{~mm}, y=20 \mathrm{~mm}$ as shown in Fig. 2(a) with the source current as defined in Fig. 5. The coupled noise waveform was obtained using Agilent ADS from the convolution of the input signal and the impulse response of the two-port network. The impulse response was generated from the inverse Fourier transform of the measured S-parameters of the test sample. As can be seen from Fig. 6(a), the maximum coupled noise for the two switching cycles is $\pm 200 \mathrm{mV}$. Noise voltage fluctuation lasts for more than $5 \mathrm{~ns}$ due to the high quality factor of the cavity structure, even after the current source has stopped the switching. Because of this long-lasting noise fluctuation, the coupled noise can be accumulated every time the current switches, which means that higher coupled noise is expected for more switching cycles. Moreover, the coupled noise is compounded by the increased magnitude of the switching current. Therefore the coupled noise through the cutout cannot be ignored, bearing in mind the tendency for rapidly increasing currents and reduced noise margins.

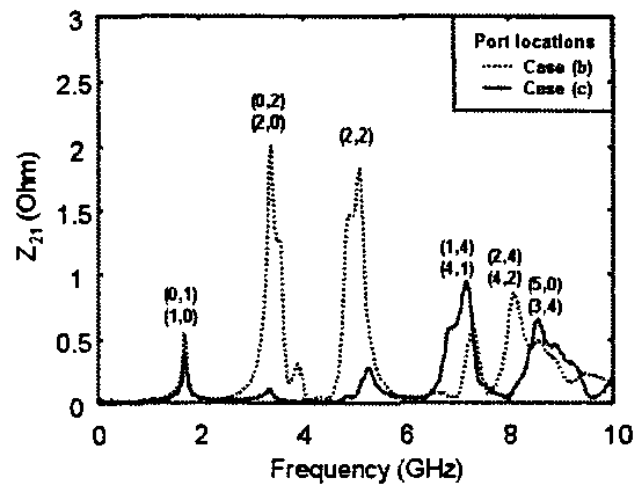

Figure 4. Measured transfer impedance: The dotted line is for the case shown in Fig. 2(b) and the solid line is for the case shown in Fig. 2(c). 


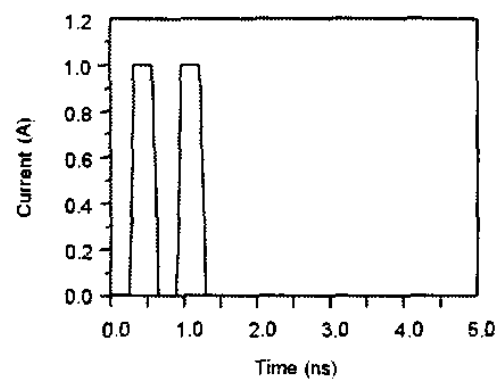

Figure 5. Assumed switching current waveform

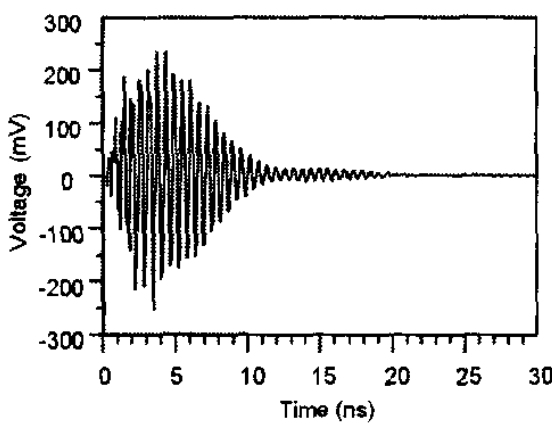

(a)

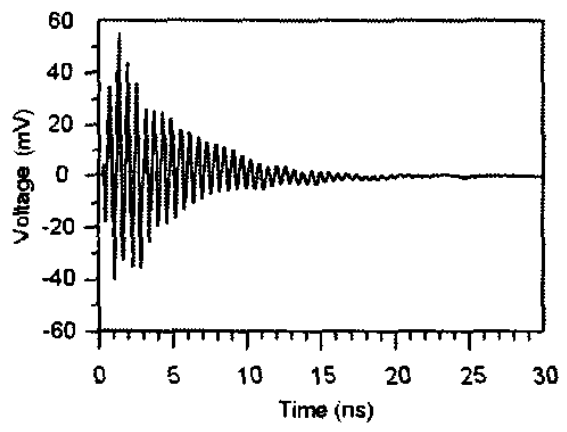

(b)

Figure 6. Time domain coupled noise waveform: (a) coupled noise waveform in the case of Fig. 2(a); (b) coupled noise waveform in the case of Fig. 2(c).

To confirm the suppression effect of the coupled noise through the cutout by repositioning chips 1 and 2, time domain transient coupled noise response for the case of Fig. 2(c) has been simulated and the reduced coupled noise is shown in Fig. 6(b). As predicted in the frequency domain simulation and measurement, the optimal positioning method is effective when applied in time domain analysis. The maximum coupled noise for the two switching cycles is $\pm 40 \mathrm{mV}$ for the case of Fig. 2(c), which is only $20 \%$ of that in the case of Fig. 2(a).

\section{THE EFFECT OF PLANE RESONANCE AND SWITCHING FREQUENCY ON COUPLED SSN THROUGH A CUTOUT}

The coupled SSN through the cutout can be expressed by the multiplication of the transfer impedance, $Z_{21}$ and switching current:

$$
V_{\text {(nuplet__sw }}(f)=Z_{21}(f) \times I_{\text {swriching }}(f)
$$

where $Z_{2 /}$ is the transfer impedance between the SSN generating port and the observation port. $I_{\text {stirching }}$ is the switching current of noise generating chip. From (1), we suppose that the coupled SSN can be suppressed when $Z_{21}$ has only low resonant peaks in the frequency range of concern as demonstrated in the section II. Alternatively, the coupled noise can be reduced if the resonant frequencies of the cavity have no correlation with the switching current.

Consider test vehicle shown in Fig. 7. The layer stack-up and the cross section of the test vehicle are the same with those shown in Fig. 1. The dimensions of it are $80 \times 80 \mathrm{~mm}^{2}$, and cutout is made in the third (Ground) layer at the coordinates of $x=55 \mathrm{~cm}$, and $y=40 \mathrm{~cm}$ with a $1 \times 20 \mathrm{~mm}^{2}$ size. The chip shown in Fig. 7 which corresponds to chipl in Fig. 1 is Texas Instruments 1:10 LVDS clock driver whose product number is CDCLVD110. Each of the nine pairs of the differential outputs is terminated to ground with $50 \mathrm{ohm}$ chip-type resistance. One pair of the output was designed to be measured to check whether the chip is working properly. Bottom layer of test vehicle which corresponds to V2 in Fig. 1 supply $2.5 \mathrm{~V}$ to the chip. There are two observation ports: one is at $(70 \mathrm{~mm}, 40$ $\mathrm{mm})$ and the other is at $(60 \mathrm{~mm}, 60 \mathrm{~mm})$ which correspond to port locations as shown in Fig. 2(b) and Fig. 2(c), respectively. Once all the ten output differential pairs switch simultaneously, it generates SSN in the bottom cavity and the SSN will coupled to the neighboring cavity through the cutout. The coupled SSN was measured at the observation ports shown in Fig. 7.

Anritsu MP1763B pulse pattern generator (PPG) was used to generate differential clock input of the chip. The coupled noise was measured using Tektronix TDS8000B digital sampling oscilloscope. To measure the noise voltage correctly, a high impedance probe has to be used. In our measurement, however, it is measured under $50 \mathrm{ohm}$ impedance condition using a SMA connector for easy probing. We believe it is valid because $50 \mathrm{ohm}$ is high enough compared with the transfer impedance, $Z_{21}$. First, we applied $900 \mathrm{MHz}$ differential clock to the input connector from PPG because it is $(1,0)$ mode resonant frequency of the test vehicle. Since the dimensions of the test vehicle are two times of those shown in Fig. 1, the resonant frequencies are half of those shown in Fig. 4.

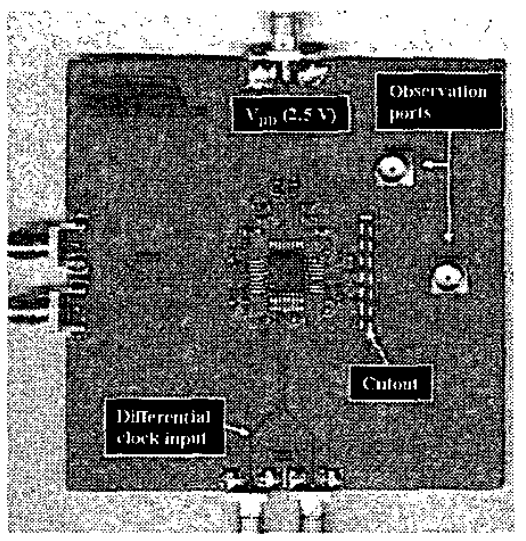

Figure 7. Photograph of test vehicle for coupled SSN measurement 


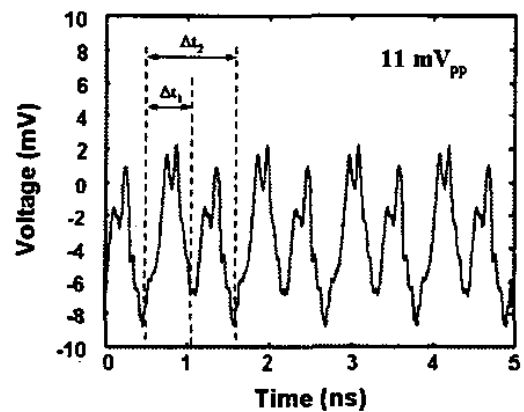

(a)

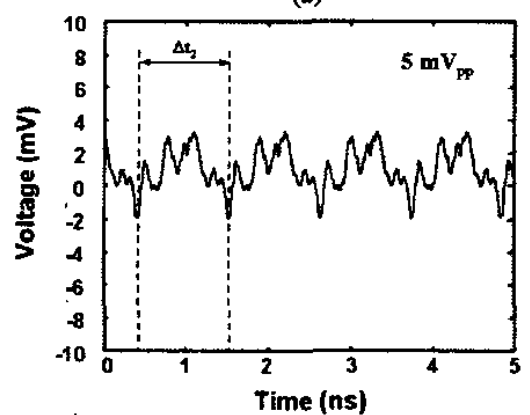

(b)

Figure 8. Measured coupled SSN when the chip switches at $900 \mathrm{MHz}$ : (a) measured at (70 mm, $40 \mathrm{~mm})$; (b) measured at $(60 \mathrm{~mm}, 60 \mathrm{~mm}) .1 / \Delta t$, and $1 / \Delta t_{2}$ are $1.8 \mathrm{GHz}$ and $900 \mathrm{MHz}$, respectively.

As can be seen in Fig. 8, the coupled noise measured at (60 $\mathrm{mm}, 60 \mathrm{~mm})$ is $5 \mathrm{mV}_{\mathrm{pp}}$ while it is $11 \mathrm{mV}_{\mathrm{pp}}$ at $(70 \mathrm{~mm}, 40 \mathrm{~mm})$. The reduction of the coupled SSN results from optimal positioning method as discussed in the previous section. $1 / \Delta t_{\text {, }}$ and $1 / \Delta t_{2}$ in Fig. 8 are $1.8 \mathrm{GHz}$ and $900 \mathrm{MHz}$, respectively. We can observe that $(2,0)$ mode whose resonant frequency is 1.8 $\mathrm{GHz}$, is dominant in Fig. 8(a) because center-positioned chip suppress $(1,0)$ modal impedance as shown in Fig. (4). In Fig. 8(b), we can see periodical waveform whose dominant frequency is $900 \mathrm{MHz}$ because of nonzero $(1,0)$ modal impedance although it is suppressed as shown in Fig. 4.

Fig. 9 shows the coupled SSN measured at $(70 \mathrm{~mm}, 40$ $\mathrm{mm}$ ) when the chip switches at $700 \mathrm{MHz}$. The coupled noise reduced to $6.5 \mathrm{mV}_{\mathrm{pp}} .(1,0)$, and $(2,0)$ modal frequencies shown in Fig. 8(a), don't appear in Fig. 9 because the switching currents don't have those frequency components such as 900 $\mathrm{MHz}$ or $1.8 \mathrm{GHz}$. From the results, we can conclude that the coupled noise can be suppressed by designing the dimensions of the cavity carefully so that the resonant frequencies of it are not related to the operating frequency of the SSN generating chip as well as using the optimal positioning method.

Fig. 10 shows the coupled SSN when the test vehicle has no cutout in the ground layer. The observation point is $(70 \mathrm{~mm}$, $40 \mathrm{~mm}$ ) and the chip switches at $900 \mathrm{MHz}$. The fluctuation is less than $2 \mathrm{mV}$ which is much smaller than that shown in Fig. 8(a). The skin depth of the copper plane is smaller than the metal thickness which results in preventing the SSN in the bottom cavity from penetrating into the neighboring cavity [5]. From the measured results shown in Fig. 8(a) and Fig. 10, we can realize that the coupled SSN through the cutout can be subs

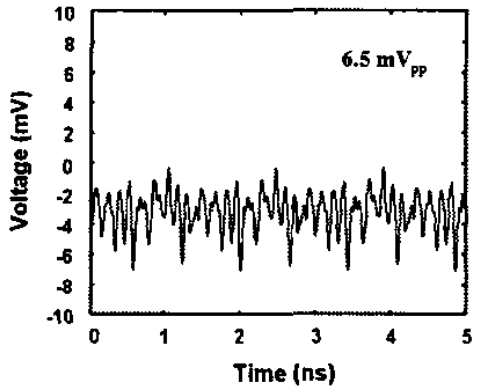

Figure 9. The coupled SSN measured at $(70 \mathrm{~mm}, 40 \mathrm{~mm})$ when the chip switches at $700 \mathrm{MHz}$

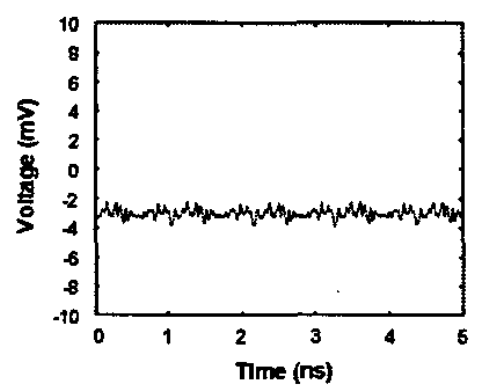

Figure 10. The coupled SSN when the test vehicle has no cutout in the ground layer. The measuring point is $(70 \mathrm{~mm}, 40 \mathrm{~mm})$ and the chip switches at 700 $\mathrm{MHz}$

-tantial and needs to be managed.

\section{CONCLUSIONS}

In this paper, we describe the reduction methods of coupled SSN through the cutout. The suppression methods were successfully demonstrated by both frequency and time domain analysis and measurement. The suppression was achieved by the suggested optimal positioning method and by changing switching frequency of the SSN generating chip. Once the operating frequency of the SSN generating chip is determined, we can design the size of plane cavity whose resonant frequencies are not related to the operating frequency of the chip.

\section{REFERENCES}

[1] R.R. Tummala, E.J. Rymaszewski, and A.G. Klopfenstein, Microelectronics Packaging Handbook Part I, New York, Chapman \& Hall, 1997, Chapter 3

[2] Junwoo Lee, Yeo Mui Seng, Mahadevan K. lyer, and Joungho Kim, "Investigation of Plane-to-Plane Noise Coupling through Cutout in Multi-layer Power/Ground Planes," Proc. of 4th Electronics Packaging Technology Conference, Singapore, pp. 257-260, Dec. 2002,.

[3] Junwoo Lee, Yeo Mui Seng, Mihai Dragos Rotaru, Mahadevan K. Iyer, and Joungho Kim, "Characterization of High-Frequency Plane-to-Plane Coupling through Cutout in Multi-Layer Packages," Proc. IEEE Electron. Comp. Technol. Conf.. pp. 1589-1593, May 2003.

[4] G. T. Lei, R. W. Techentin, and B.K Gilbert, "High-Frequency Characterization of Power/Ground-Plane Structures", IEEE Trans. Microwave Theory Tech., vol. 47, no. 5, pp. 562-569, May 1999.

[5] J. Mao, J. Srinivasan, J. Choi, M. Swaminathan, and N. Do, "Modeling of field penetration through planes in multilayered packages", IEEE Trans. Advanced Packaging, vol 24, no. 3, pp. 326-333, Aug. 2001. 